\title{
Satisfação no Trabalho na Percepção de Gestores Escolares de Escolas Públicas Municipais
}

\author{
Satisfaction in the Work in the School Managers' Perception of Municipal Public Schools
}

\author{
Alda Dantas do Rêgo ${ }^{a}$; Iracema Rocha da Silva ${ }^{\mathrm{a}}$; Adriana Leônidas de Oliveira*b \\ ${ }^{a}$ Universidade Federal do Maranhão, MA, Brasil. \\ •Universidade de Taubaté: Programa de Pós-Graduação Stricto Sensu em Administração. SP, Brasil. \\ *E-mail: adrianaleonidas@uol.com
}

\begin{abstract}
Resumo
Qualidade de Vida no Trabalho (QVT) é termo que traduz a ideia de bem-estar e propõe melhorias nas condições do ambiente de trabalho. Este artigo é parte dos achados da dissertação desenvolvida no Programa de Pós-Graduação em Administração, que teve por objetivo caracterizar a Qualidade de Vida no Trabalho de gestores de escolas públicas da rede municipal de ensino da cidade de Imperatriz, Estado do Maranhão, bem como sua satisfação com o trabalho. Trata-se de estudo descritivo, com abordagem predominantemente quantitativa, com aplicação de questionário fechado. A amostra foi definida por acessibilidade, constituída de 119 gestores, obtendo 86 respondentes. Os resultados indicaram que há um sentimento de satisfação para a maioria dos investigados com relação a sua QVT. Destaque de satisfação para as variáveis: importância do trabalho para a sua vida, valorização de sua ideia e iniciativa no ambiente de trabalho, orgulho de realizar seu trabalho e relacionamento entre colegas e chefias, o que propicia uma interferência considerada positiva na gestão escolar. Todavia, houve um descontentamento acentuado com relação à variável remuneração $(35,3 \%)$; porém, $46,5 \%$ dos gestores apresentaram sentimento de indiferença relacionado aos benefícios extras recebidos e $32,6 \%$ com relação aos equipamentos de segurança. Pode-se concluir que apenas $2,3 \%$ dos entrevistados relataram insatisfação, enquanto $81,4 \%$ expressaram satisfação, seguidos de $16,3 \%$, que se encontram muito satisfeitos com o que fazem. Portanto, os gestores que constituíram a amostra demonstraram satisfação quanto a sua Qualidade de Vida no Trabalho.
\end{abstract}

Palavras-chave: Qualidade de Vida no Trabalho. Satisfação. Gestores Escolares.

\begin{abstract}
Quality of Life at Work, term that makes the idea of well-being and proposes improvements in conditions in the work environment. This article is part of the findings of the dissertation developed in the Graduate Program in Administration, which aimed to characterize the Quality of Life in the Work of public school managers of the municipal education network of the city of Imperatriz, state of Maranhão, as well as their satisfaction with the job. This is a descriptive study, with a predominantly quantitative approach, with the application of a closed questionnaire. The sample was defined by accessibility, consisting of 119 managers, obtaining 86 respondents. The results indicated that there is a satisfaction feeling for the majority of respondents regarding their QWL. Highlighting satisfaction for the variables: work importance for their life, valorization of their idea and initiative in the work environment, pride in performing their work and relationship between colleagues and managers, which leads to interference considered positive in school management. However, there was a marked dissatisfaction with the variable remuneration $35.3 \%$; but $46.5 \%$ of the managers presented a feeling of indifference related to the extra benefits received and $32.6 \%$ regarding safety equipment. It can be concluded that only 2.3\% of the respondents reported dissatisfaction, while $81.4 \%$ expressed satisfaction, followed by $16.3 \%$ who are very satisfied with what they do. Therefore, the managers who constituted the sample showed satisfaction with their Quality of Life at Work.
\end{abstract}

Keywords: Quality of Life at Work. Satisfaction. School Managers.

\section{Introdução}

Desde o início do processo histórico da Qualidade de Vida no Trabalho (QVT), em 1920, se têm valorizado fatores como a satisfação e o bem-estar do trabalhador como forma de melhoria no ambiente laboral. Dessa forma, o tema ganhou relevância significativa tanto para o trabalhador no ambiente de trabalho, quanto para gestão da organização (LIMONGI FRANÇA, 2014).

AQVT se caracteriza por ações que buscam a humanização das relações de trabalho e que mantenham uma estreita ligação entre a satisfação do indivíduo no ambiente laboral e a produtividade da organização/instituição.

Essas ações decorrem do elevado nível de exigência das organizações pela apresentação de resultados, pela amplitude da concorrência e pelo nível de complexidade exigido para que estas se mantenham ativas no mercado.

O presente artigo é parte de uma dissertação, que abordou a caracterização da Qualidade de Vida no Trabalho de gestores escolares, baseada no modelo proposto por Walton (1973), tendo como campo de aplicação e objeto de pesquisa gestores de escolas públicas. As variáveis permitiram mensurar o nível de satisfação da população investigada no que diz respeito à remuneração, às condições e jornadas de adequação ao trabalhador, além de analisar as oportunidades presentes e futuras e o aproveitamento de potencialidades dos gestores.

Com os resultados alcançados foi possível fornecer informações importantes para subsidiar, tanto a gestão pública 
municipal, quanto a política administrativa, e possibilitar sugestões de melhorias na QVT dos gestores de escolas públicas municipais.

\section{Material e Métodos}

\subsection{Delineamento}

Trata-se de uma pesquisa descritiva com abordagem quantitativa, essa metodologia tem como objetivo descrever determinado fenômeno. Sob essa ótica, quando se pretende obter informações sobre determinado grupo ou indivíduo se utiliza, também, o método descritivo.

O delineamento foi realizado por meio de levantamento, já que mensura os resultados, aprimorados com o caráter descritivo da pesquisa. A população pesquisada, definida por acessibilidade, foi constituída por 119 gestores escolares no universo amostral, obtendo 86 respondentes. Quanto a sua área de realização, foram escolhidas as escolas públicas municipais (Educação Infantil e Ensino Fundamental).

Foram utilizados dois instrumentos, um sociodemográfico e outro sobre a QVT, aplicados individualmente (visita in loco) a cada pesquisado após aprovação do Comitê de Ética da UNITAU. Os dados foram analisados, tabulados e organizados por meio de gráficos e tabelas, com o auxílio do Excel e do software SPSS.

\subsection{Estudos sobre a Qualidade de Vida no Trabalho na Área da Educação}

Considerando as buscas empreendidas, foi possível assinalar que, no Brasil, o tema deste estudo (Qualidade de Vida no Trabalho na área da Educação) ainda carece de pesquisas. Entretanto, identificaram-se ensaios que tratam isoladamente de profissionais da área da Educação brasileira, inclusive, relacionando-os a diversas outras categorias, como estresse, satisfação no trabalho, entre muitos outros.

Visando o levantamento de trabalhos sobre o assunto, inicialmente, foram realizadas buscas sobre a qualidade de vida no trabalho de gestores de escolas públicas da Educação Básica. Como não foram encontradas pesquisas específicas, tiveram início diligências no portal eletrônico IBICT, SciELO, periódicos de Psicologia e Google Acadêmico, com o intuito de identificar estudos que tratassem a QVT associada a esta categoria.

Segundo os levantamentos feitos por Santana et al. (2012), que buscaram investigar a satisfação no trabalho de gestores de escolas da Educação Básica de 52 cidades do Estado de Minas Gerais, observa-se que há satisfação na gestão escolar por partes dos gestores. Para isso, os autores arrolaram um instrumento de pesquisa que possibilitou a investigação do fenômeno satisfatório da qualidade de vida no trabalho a partir de referenciais teóricos, constatando-se a adequação da utilização dos indicadores de QVT, proposto por Walton (1973).
Moreira et al. (2010) analisam a relação da QVT e o estilo de vida considerando os ciclos vitais dos professores de Educação Física. O objetivo deste estudo foi analisar a percepção da qualidade de vida no trabalho e o perfil do estilo de vida, considerando os ciclos vitais desses profissionais pertencentes à rede pública estadual de ensino do Paraná. Participaram da pesquisa 654 professores. O instrumento utilizado foi uma Escala de Avaliação da QVT dos professores desenvolvido a partir das oito Dimensões propostas por Walton (1973).

Corroborando com esse estudo, Moreira et al. (2010) ainda investigaram a relação da QVT com o estilo de vida associado aos ciclos de desenvolvimento profissional com a mesma população pesquisada. $\mathrm{Na}$ análise estatística se utilizou o qui-quadrado para o grupo amostral. Os resultados indicaram que a maioria dos pesquisados estão satisfeitos com a QVT, levando os autores à conclusão de que, com o avanço na carreira, aumenta a insatisfação com o desenvolvimento profissional.

Quanto aos aspectos da QVT dos professores entrevistados, ambas as pesquisas apresentaram avanços significativos sobre a qualidade de vida no trabalho dos profissionais. Moreira et al. (2009) também investigaram a QVT e a síndrome de Burnout em uma amostra de 146 professores de Educação Física do Estado do Rio Grande do Sul. Na análise dos dados, foram utilizados o teste qui-quadrado e o teste de correlação de Spearman.

Schuch (2001) avaliou os fatores determinantes da QVT de docentes da Universidade do Planalto Catarinense (Uniplac) baseado no modelo proposto por Walton (1973) para análise da percepção dos profissionais participantes, e procurou definir o grau de satisfação dos professores com o trabalho. Esse estudo teve como objetivo analisar como se caracteriza, no entendimento dos professores da Uniplac, a qualidade de vida no trabalho e se configurou como estudo de caso do tipo descritivo-analítico, com a aplicação de um questionário a 102 professores, com a utilização da escala Likert composta por cinco valores. Sobre essa pesquisa, o autor concluiu que, dos oito fatores analisados, quatro foram considerados razoáveis para os entrevistados, enquanto os outros fatores receberam avaliação de "pouco satisfeitos". Com isso, a QVT do grupo pesquisado, de forma geral, é regular e requer melhorias por meio de investimentos e da implantação de um programa de desenvolvimento gerencial.

Na investigação realizada por Mendonça (2013) se buscou analisar o nível de QVT, conforme a concepção do docente efetivo do Ensino Superior do Centro Socioeconômico da Universidade Federal de Santa Catarina (UFSC). Foram avaliados 146 docentes pertencentes aos três Departamentos da Universidade e o procedimento adotado foi a abordagem quali-quantitativa. A pesquisa foi considerada um estudo de caso, de natureza exploratória e descritiva. O instrumento 
utilizado, um questionário estruturado e semiaberto, desenvolvido pelo próprio autor, baseado na percepção dos docentes, permitiu concluir que o investimento na QVT do serviço público proporciona melhorias às pessoas e aos profissionais envolvidos, e sugerir a implantação de um programa de Qualidade de Vida no Trabalho, que valorize a profissão dos docentes.

Silva (2002) analisou as variáveis da QVT relacionadas ao estresse e à saúde dos professores universitários. O estudo, que contou com a participação de 170 professores Universitários (privados e públicos) da cidade de Belo Horizonte, MG, foi descritivo e comparativo, com foco na abordagem qualitativa por meio de survey. O método utilizado foi a entrevista semiestruturada e a observação da pesquisa. Os resultados encontrados associam o alto nível de estresse à insatisfação no trabalho, e o autor recomendou a reconstrução das relações entre trabalho e profissão da docência, a fim de promover o desenvolvimento humanizado do trabalho docente.

O estudo de Petroski (2005) abordou a QVT e suas relações com o estresse de professores universitários, com o propósito de avaliar, na percepção dos professores, a QVT, o estresse, o nível de atividade física e o risco coronário dos professores da UFSC. A amostra analisada foi de 1.420 professores, que foram submetidos a um questionário, desenvolvido pelo autor, a partir das Dimensões propostas por Walton (1973), com o intuito de avaliar sua QVT.

Como resultado se observou que, quanto mais desenvolvida a QVT, menor o nível de estresse dos professores. Analisando a pesquisa empreendida se percebe a tendência do tipo quanti-qualitativa (mista) para mensuração da QVT, e em cinco, das nove publicações, os autores utilizaram o modelo proposto por Walton (1973) como instrumento de avaliação da QVT dos grupos investigados. O público investigado foi integralmente composto por profissionais da Educação (professores), pertencentes, em sua maioria, ao Ensino Superior de Universidades públicas federais, localizadas nas regiões Sul e Sudeste do Brasil.

De maneira geral, os estudos sobre a Qualidade de Vida no trabalho têm mostrado resultados e conclusões satisfatórias. Entretanto, os profissionais da Educação sofrem com as constantes mudanças impostas pelo mundo atual, que culminam com o desgaste no trabalho, gerando problemas que vão desde a saúde física e mental até a falta de qualificação e valorização financeira e social.

\section{Resultados e Discussão}

Quanto à caracterização da QVT dos gestores escolares se utilizou um instrumento padronizado, segundo modelo proposto por Walton (1973), contemplando as seguintes dimensões: compensação justa e adequada, condições de trabalho e desenvolvimento de capacidades; oportunidades de crescimento e segurança; integração social na organização; constitucionalismo; o trabalho e o espaço total na vida do indivíduo; e relevância social, adaptadas por Fernandes (1996).

\subsection{Dimensões de satisfação da Qualidade de Vida no Trabalho}

Na avaliação da QVT dos gestores, a dimensão Uso de suas capacidades no trabalho apresentou o maior percentual de satisfação (59,3\%), com destaque para as variáveis: (a) a importância das atividades e (b) a responsabilidade conferida, com indicadores de maior satisfação. As informações convergem, coerentemente, com os dados das pesquisas realizadas por Santana et al. (2012) e Mendonça (2013), em percentuais aproximados nos resultados, o que propicia uma presença de satisfação entre os pesquisados.

Os resultados afetos à dimensão Oportunidade de crescimento no trabalho foram considerados satisfatórios, assinalando um sentimento de contentamento entre os pesquisados, uma vez que $36,8 \%$ se demonstraram satisfeitos. Há um sentimento e uma expectativa de melhorias relacionadas à oportunidade de crescimento e à tendência para satisfação.

Quanto à dimensão Integração social na organização, para os investigados, a integração social na percepção foi satisfatória $(60,2 \%)$, enquanto $19,1 \%$ se posicionaram de forma indiferente e apenas $6,8 \%$ se revelaram insatisfeitos. Entre as variáveis desta dimensão, os entrevistados apresentaram percentual elevado ao sentimento de "satisfação".

Entretanto, na dimensão constitucionalismo, 45,7\% dos investigados se sentiam satisfeitos; em contrapartida, em um contexto geral, 32,9\% apresentaram sentimentos de indiferença com relação à temática.

No tocante aos resultados apontados pela dimensão "O trabalho e o espaço total na vida" se percebe que houve um equilíbrio entre o trabalho e o espaço da vida dos indivíduos, embora exista uma tendência considerável de investigados, que se sentem indiferentes com relação a este indicador; $41,6 \%$ afirmaram satisfação com a influência do trabalho em suas vidas pessoais e tendência de maior satisfação para a variável influência do trabalho na vida e rotina familiar.

Nesse cenário, Sant'Anna, Kilimnik e Moraes (2011) ressaltam que o trabalho não deve ocupar todo o tempo da vida do indivíduo, sendo necessário criar um espaço de lazer e de convivência em comunidade, buscando um ponto de equilíbrio entre trabalho e vida pessoal.

A dimensão Relevância social do trabalho indica que $50,3 \%$ dos investigados se encontram satisfeitos e $17,7 \%$ se acham muito satisfeitos. O Quadro 1 ilustra a consolidação das dimensões de satisfação da QVT da população entrevistada. 
Quadro 1 - Dimensões de satisfação da Qualidade de Vida no Trabalho

\begin{tabular}{|c|c|c|c|c|c|}
\hline \multirow[b]{2}{*}{ Dimensão } & \multicolumn{5}{|c|}{ QVT dos gestores } \\
\hline & 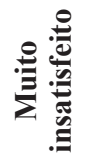 & 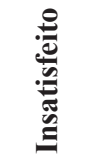 & 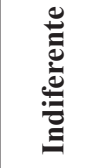 & 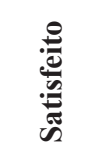 & 兑 \\
\hline $\begin{array}{l}\text { 1. Uso e } \\
\text { desenvolvimento de } \\
\text { capacidades }\end{array}$ & $1,4 \%$ & $7,4 \%$ & $20,9 \%$ & $59,3 \%$ & $10,9 \%$ \\
\hline $\begin{array}{l}\text { 2. Oportunidade } \\
\text { de crescimento e } \\
\text { segurança }\end{array}$ & $4,3 \%$ & $13,6 \%$ & $31,0 \%$ & $36,8 \%$ & $14,3 \%$ \\
\hline $\begin{array}{l}\text { 3. Integração social } \\
\text { na organização }\end{array}$ & $1,6 \%$ & $6,9 \%$ & $11,7 \%$ & $61,2 \%$ & $18,6 \%$ \\
\hline $\begin{array}{l}4 . \\
\text { Constitucionalismo }\end{array}$ & $3,9 \%$ & $9,7 \%$ & $32,9 \%$ & $45,7 \%$ & $7,8 \%$ \\
\hline $\begin{array}{l}\text { 5. O trabalho e o } \\
\text { espaço total da vida }\end{array}$ & $2,1 \%$ & $10,3 \%$ & $34,2 \%$ & $41,6 \%$ & $11,9 \%$ \\
\hline $\begin{array}{l}\text { 6. Relevância social } \\
\text { do trabalho na vida }\end{array}$ & $3,2 \%$ & $6,1 \%$ & $22,7 \%$ & $50,3 \%$ & $17,7 \%$ \\
\hline $\begin{array}{l}\text { Avaliação geral da } \\
\text { QVT }\end{array}$ & $2,3 \%$ & $6,8 \%$ & $21,8 \%$ & $54,8 \%$ & $14,3 \%$ \\
\hline
\end{tabular}

Fonte: dados da pesquisa.

\subsection{Dimensões de insatisfação da Qualidade de Vida no Trabalho}

A dimensão da QVT intitulada Compensação justa e adequada foi avaliada por meio da satisfação com itens relativos à remuneração, benefícios extras, entre outros. Todas as variáveis analisadas revelaram que os pesquisados se sentem entre muito insatisfeitos (13,2\%), insatisfeitos $(35,3 \%)$ ou nem satisfeitos, nem insatisfeitos $(37,6 \%)$, e que discordam da política salarial na qual estão inseridos.

Neste contexto, os investigados afirmaram descontentamento com relação a esta dimensão, com posicionamentos fundamentados no sentimento de injustiça por considerarem que seus proventos são baixos ou menores, se comparados com o salário de colegas que desenvolvem as mesmas atividades e ganham mais. A variável salário é um indicador que deve ser alvo de melhorias dentro das instituições por se tratar de um fator de descontentamento profissional, conforme apontamentos de outras pesquisas realizadas por Santana et al. (2012), Rosa (2012), Sant'Anna e Kilimnik e Moraes (2011).

A dimensão Condições de trabalho está relacionada à satisfação com as condições e jornada de trabalho e ao uso de tecnologias, equipamentos de segurança e cansaço causado pelo trabalho. Com relação às condições de trabalho se constatou que os investigados tendem à insatisfação, pois $61,9 \%$ se revelaram desde muito insatisfeitos até indiferentes, muito embora a avaliação geral conclua pela satisfação entre a maioria simples dos entrevistados (35,8\%). O Quadro 2 apresenta um resumo consolidado das dimensões insatisfatórias da QVT.
Quadro 2 - Dimensões de insatisfação da Qualidade de Vida no Trabalho

\begin{tabular}{|c|c|c|c|c|c|}
\hline \multirow[b]{2}{*}{ Dimensão } & \multicolumn{5}{|c|}{ QVT dos gestores } \\
\hline & 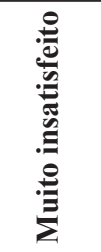 & 咆 & & 窇 & 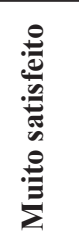 \\
\hline $\begin{array}{l}\text { 1. Compensação } \\
\text { justa e adequada }\end{array}$ & $13,2 \%$ & $35,3 \%$ & $37,6 \%$ & $14,0 \%$ & $0,0 \%$ \\
\hline $\begin{array}{l}\text { 2. Condições de } \\
\text { trabalho }\end{array}$ & $7,9 \%$ & $22,1 \%$ & $31,9 \%$ & $35,8 \%$ & $2,3 \%$ \\
\hline $\begin{array}{l}\text { Avaliação geral } \\
\text { da QVT }\end{array}$ & $10,5 \%$ & $28,7 \%$ & $34,8 \%$ & $24,9 \%$ & $1,2 \%$ \\
\hline
\end{tabular}

Com base no modelo de Walton (1973) se evidenciou que os gestores que constituíram a amostra demonstraram satisfação quanto a sua Qualidade de Vida no Trabalho, conforme ilustrado no Gráfico 1, que aborda as oito dimensões desse modelo de forma conjunta.

\section{Conclusão}

Considerando a Qualidade de Vida no Trabalho da população pesquisada, em linha gerais, apenas $2,3 \%$ relataram insatisfação, enquanto $81,4 \%$ manifestaram satisfação, seguidos de $16,3 \%$ que se encontram muito satisfeitos com o que fazem. Dessa forma, a satisfação com a QVT, na perspectiva dos gestores de escolas públicas municipais, ainda enfrenta grandes desafios e peculiaridades em função da complexidade e à centralidade das instituições. Ainda de acordo com os resultados, há uma predominância do gênero feminino (89,5\%) na população investigada, em que $80,2 \%$ possuem especialização e a média de idade gira entre 30 e 45 anos. Os gestores que constituíram a amostra demonstraram satisfação com relação a sua QVT.

Apesar de a maioria dos investigados apresentar satisfação quanto a sua Qualidade de Vida no Trabalho, há variáveis que requerem atenção especial: fator salarial, uso de tecnologia no ambiente de trabalho, equipamentos de segurança e o incentivo que a instituição oferece para a continuidade dos estudos. Portanto, diante da relevância deste trabalho e, conforme os resultados apurados na pesquisa, se conclui que seu objetivo geral, que trata de caracterizar a Qualidade de Vida no Trabalho de gestores de escolas públicas municipais do município de Imperatriz, Estado do Maranhão, foi atingido, bem como os seus objetivos específicos.

Diante do exposto, ao finalizar a investigação da literatura utilizada como fundamentação do estudo e a aplicação e análise da pesquisa realizada junto aos gestores escolares se pode concluir que a Qualidade de Vida no Trabalho (QVT) se apresenta como sendo um grande desafio diário, e que a implementação de ações de QVT no ambiente de trabalho não é tarefa fácil. 


\section{Referências}

FERNANDES, E.C. Qualidade de vida no trabalho: como medir para melhorar. Salvador: Casa da Qualidade, 1996.

LIMONGI FRANÇA, A.C. Qualidade de vida no trabalho: conceitos e práticas nas empresas da sociedade pós-industrial. São Paulo: Atlas, 2014.

MENDONÇA, M.H. A qualidade de vida no trabalho na Universidade Federal de Santa Catarina: um estudo de caso com os docentes efetivos de ensino superior do Centro Socioeconômico. Florianópolis: Universidade Federal de Santa Catarina, 2013.

MOREIRA, H.R. et al. Qualidade de vida do trabalhador docente e os ciclos vitais de professores de Educação Física do Estado do Paraná, Brasil. Rev. Bras. Ciênc. Mov., p.12-20, 2010

PETROSKI, E.C. Qualidade de vida no trabalho e suas relações com estresse, nivel de atividade física e risco coronariano de professores universitários. Florianópolis: UFSC, 2005.
ROSA, S.J. A qualidade de vida no trabalho dos professores do ensino médio da educação básica pública e privada de Paracatu - MG. Pedro Leopoldo: FPL, 2012.

SANT'ANNA, A.S.; KILIMNIK, Z.M.; MORAES, L.F.R. Antecedentes, origens e evolução do movimento em torno da Qualidade de Vida no Trabalho. Qualidade de vida no trabalho: abordagens e fundamentos. Rio de Janeiro: Elsevier, 2011.

SANTANA, L.L.S. et al. Gestores escolares da rede pública em Minas Gerais: fatores de satisfação no trabalho. Educ. Rev., v.28, n.1, p.491-520, 2012.

SCHUCH, C. Qualidade de vida no trabalho: a percepção dos professores da Universidade do Planalto Catarinense. Florianópolis: Universidade Federal de Santa Catarina, 2001.

SILVA, M.A.L. Qualidade de vida no trabalho, estresse e saúde mental dos professores universitários: um estudo comparativo entre instituições públicas e privadas em Belo Horizonte - MG. Florianópolis, São Paulo: UFSC, 2002. 